\title{
LSPR Chip for Parallel, Rapid, and Sensitive Detection of Cancer Markers in Serum
}

\author{
Srdjan S. Aćimović, ${ }^{\dagger}$ Maria A. Ortega, ${ }^{\dagger}$ Vanesa Sanz, ${ }^{\dagger}$ Johann Berthelot, ${ }^{\dagger}$ Jose L. Garcia-Cordero, ${ }^{\dagger}$ \\ Jan Renger, ${ }^{\dagger}$ Sebastian J. Maerkl, ${ }^{\dagger}$ Mark P. Kreuzer, ${ }^{* \dagger}$ and Romain Quidant ${ }^{*}, \dagger, \S$ \\ ${ }^{\dagger}$ ICFO - Institut de Ciencies Fotoniques, Mediterranean Technology Park, 08860 Castelldefels (Barcelona), Spain \\ ${ }^{\ddagger}$ Institute of Bioengineering, School of Engineering, École Polytechnique Fédérale de Lausanne (EPFL), Route Cantonale, 1015 \\ Lausanne, Switzerland \\ ${ }^{\S}$ ICREA - Institució Catalana de Recerca i Estudis Avançats, Passeig de Lluís Companys, 23, 08010 Barcelona, Spain
}

Supporting Information

ABSTRACT: Label-free biosensing based on metallic nanoparticles supporting localized surface plasmon resonances (LSPR) has recently received growing interest (Anker, J. N., et al. Nat. Mater. 2008, 7, 442-453). Besides its competitive sensitivity (Yonzon, C. R., et al. J. Am. Chem. Soc. 2004, 126, 12669-12676; Svendendahl, M., et al. Nano Lett. 2009, 9, 4428-4433) when compared to the surface plasmon resonance (SPR) approach based on extended metal films, LSPR biosensing features a high-end miniaturization potential and a significant reduction of the interrogation device bulkiness, positioning itself as a promising candidate for point-of-care diagnostic and field applications. Here, we present the first, paralleled LSPR lab-on-a-chip realization

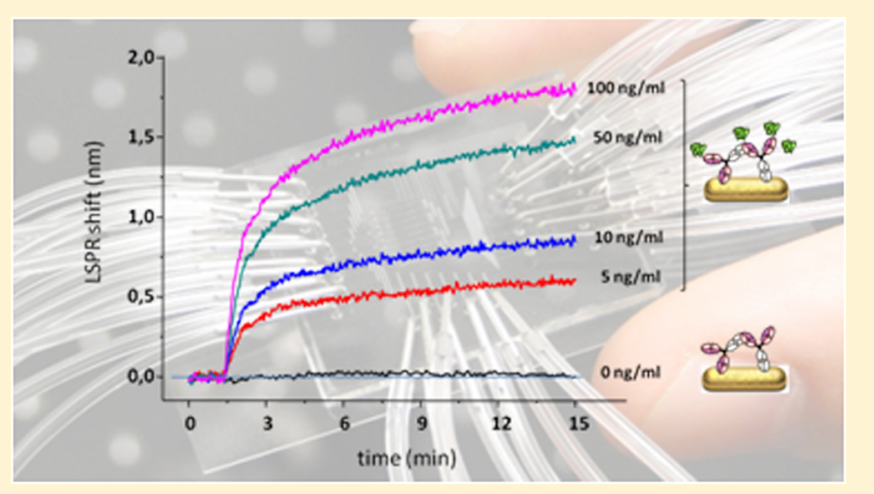
that goes well beyond the state-of-the-art, by uniting the latest advances in plasmonics, nanofabrication, microfluidics, and surface chemistry. Our system offers parallel, real-time inspection of 32 sensing sites distributed across 8 independent microfluidic channels with very high reproducibility/repeatability. This enables us to test various sensing strategies for the detection of biomolecules. In particular we demonstrate the fast detection of relevant cancer biomarkers (human alpha-feto-protein and prostate specific antigen) down to concentrations of $500 \mathrm{pg} / \mathrm{mL}$ in a complex matrix consisting of $50 \%$ human serum.

KEYWORDS: Plasmonics, LSPR, parallel, biosensing, lab-on-a-chip, cancer

$\mathrm{T}^{\mathrm{h}}$ he biosensing community has long been striving for an idealistic device consisting of high sensitivity, specificity, selectivity, and parallel real-time detection, coupled with low production and operational costs. In addition this device should be both environmentally and user-friendly and be portable, robust, and resistant to a wide range of external conditions (temperature, electromagnetic (EM) radiation, humidity), among other things. On the road toward this biosensing "Holy Grail", contemporary technology has been able to deliver numerous classes of biosensors that are focused on a particular application or niche in the biosensing market; however, no such device currently exists that delivers all or most of these requirements. Among these, optical biosensors operating in a label-free format have positioned themselves as very promising candidates owing to the inherent properties of light. Speed, inertness to external interferences, and almost unlimited bandwidth for data transfer has made light a preferred choice of transduction. With the advent of nanotechnology, especially in the field of plasmonics, surface plasmon resonance (SPR) technology revolutionized the biosensing field in the last two decades. The gold standard status of SPR is owed largely to its highly sensitive transducing mechanism. Namely, surface propagating EM waves called surface plasmon polaritons (SPPs) exhibit extraordinary sensitivity to the refractive index interfacial changes at the boundary between metal and dielectric. However, the activation of this transducing mechanism requires rather complex supporting optics, due to the inability of SPP excitation by freely propagating light. ${ }^{4}$ Moreover, already involving sophisticated optics, the expansion toward parallel, real-time mode of operation showed in most of the cases the inverse relation between number of detection channels and the device overall performance (e.g., signal-tonoise and limit of detection), per se. ${ }^{5}$ These are the main factors that inhibit straightforward implementation of SPR for in-field applications (out-of-the-lab), especially when it comes to sampling of very complex media (e.g., body fluids). Therefore, to overcome the miniaturization limit of $100 \mu \mathrm{m}^{2}$ due to the propagating nature of SPPs and device bulkiness,

Received: February 6, 2014

Revised: April 4, 2014

Published: April 14, 2014 

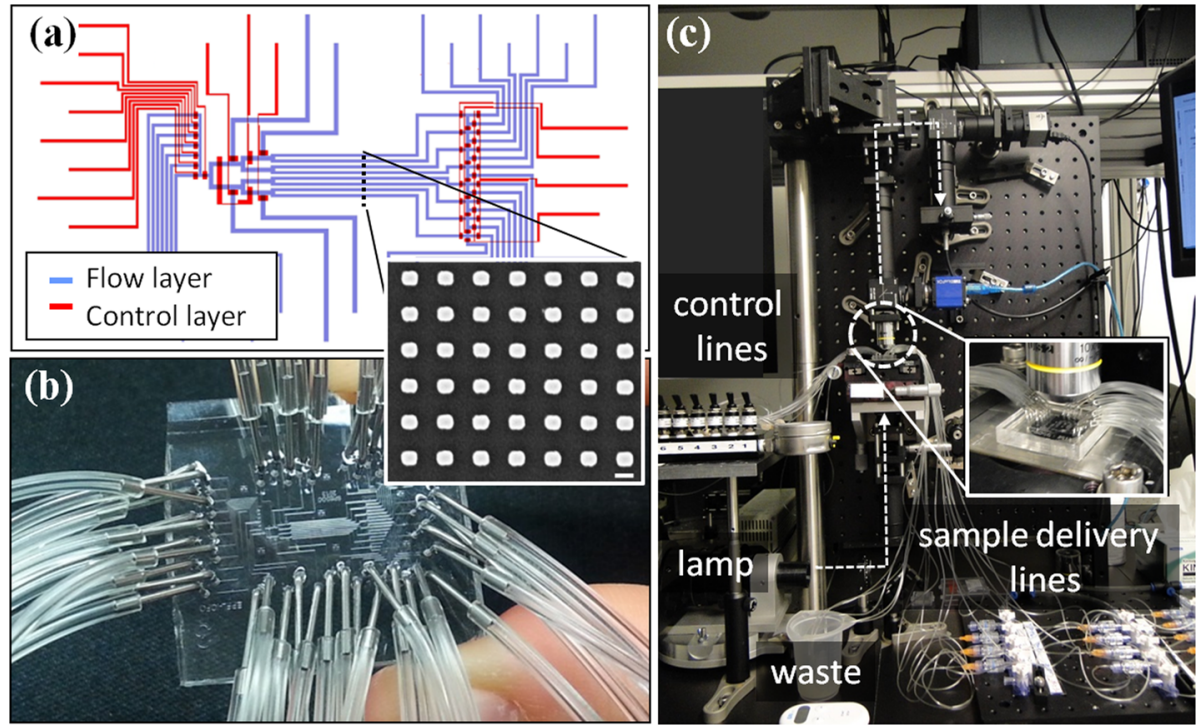

Figure 1. Description of the sensing platform: Schematic of the flow and control layers consisting the microfluidic chip (a) and final connected chip (b). The inset shows a standard SEM image of the plasmonic gold sensors. Scale bar $=200 \mathrm{~nm}$. (c) Overview of the optical setup.

metallic nanoparticles supporting localized surface plasmon resonances (LSPR) have been initially identified as the next generation of optical label-free biosensors mainly due to the extreme sensor miniaturization to the scale of a single nanoparticle. Just recently, single nanoparticle sensors have been run in the multiplex scheme and are capable of resolving even single protein binding events. ${ }^{6-10}$ Nevertheless, from the practical point of view, there is a consensus that nanoparticle ensemble schemes are a more appropriate biosensing format. ${ }^{11}$ Intrinsically involved spatial averaging by tracking an ensemble instead of isolated nanoparticle, at least from the statistical point of view, contributes to biological relevance of the acquired data, together with the relaxed constraints of nanoparticle morphological variations, simplified optics, faster data acquisition, and improved signal to noise ratio. Coupled to the inherent property of direct excitation of LSPR with freely propagating light relaxes significantly the complexity of the optics in comparison to SPR. The resonant optical signature of nanoparticle ensembles (substrate confined or free in solution) is utilized to enhance the read-out in what is called a plasmonic enzyme-linked immunosorbent assay (ELISA). ${ }^{12-15}$ Finally, the strong field confinement in metallic nanostructures renders metallic nanoparticles advantageous when considering shallow refractive index changes as in the examples of biomolecular detection $^{2,3}$ and eliminates the need for a typical thermal stabilization as in the SPR case. While there are obvious upsides, one final consideration must be given to the substrate nanostructuring cost, which initially was an inhibition in relation to commercial success. However, this cost is constantly dropping, as new parallel nanofabrication procedures are emerging and thus overcoming the cost-ineffectiveness of electron beam lithography and focused ion-beam approaches. ${ }^{16-18}$ While prior efforts of LSPR biosensing development were focused toward identifying the optimum nanoparticle configuration for maximum detection sensitivity (nanoparticle pairs, ${ }^{19,20}$ Fano-resonance supporting particles, $^{21,22}$ nanoshells, ${ }^{23,24}$ flow-through nanoholes ${ }^{25-27}$ ), real life applications required an advanced liquid sample handling interface compatible with the peculiarity of the detection principle. Actually, the realization of a competitive LSPR biosensing system stalled, mainly due to the high degree of complexity involved. Here, we demonstrate the first generation of a parallel LSPR biosensing platform with increased throughput, speed, and real-time analysis that can be almost immediately transferable to a clinical or pharmaceutical environment.

In this specific implementation, we focus on a simple periodic arrangement of gold nanorods immobilized on a glass substrate in line with the demonstration by Chen et al. ${ }^{28}$ Figure 1 (inset) presents a scanning electron microscopy (SEM) image of a portion of a typical nanorod array. A detailed description of the nanofabrication procedure is given in the Supporting Information. To ensure the independence of all nanoparticle arrays, precise sample delivery and isolation among arrays is achieved by a polydimethoxysiloxane (PDMS) polymer-based microfluidic interface actively controlled by micromechanical valves (MMV)..$^{29,30}$ The active control allows switching the microfluidic network function between various modes of operation that will be used during the different steps in the sensor preparations and sample interrogation. Additional information about the PDMS device construction and operational principles can be found in the Supporting Information and refs 29, 30, and 37. Finally, once the PDMS chip is prepared, it is aligned over the plasmonic glass substrate, where distributed nanoparticle arrays coincide within predetermined regions inside 8 individual channels (Figure 1a). Once the firm bond between PDMS and glass substrate is obtained, the device is ready to be mounted and connected to the controlled, liquid delivery module as shown in Figure $1 \mathrm{~b}$.

The optical setup used to monitor the chip consists of a homemade microscope in a bright-field transmission configuration equipped with scanning detection combined with a visible-near-infrared (VIS-NIR) light source and a spectrometer (Figure 1c). The spectrometer, cameras, and the scanning system are controlled via an in house, developed Labview interface, which also handles data acquisition, analysis, and the real-time display of the results. The system performs extinction peak and centroid tracking ${ }^{31,32}$ of distributed sensors at a sampling rate of $10 \mathrm{~Hz}$ limited by the current read-out time of 
(a)

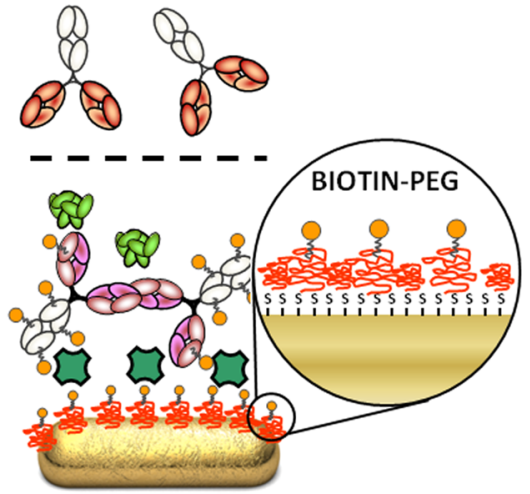

avidin

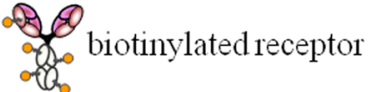

88 receptor (b)
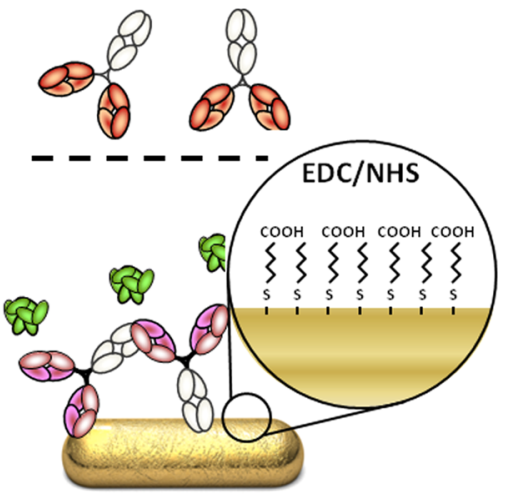

antigen

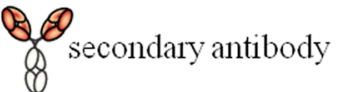

Figure 2. Schematic of the sensing approaches used for the detection of the analyte of interest where (a) a biotin-avidin is used to link the receptor and (b) carbodiimide chemistry is used.

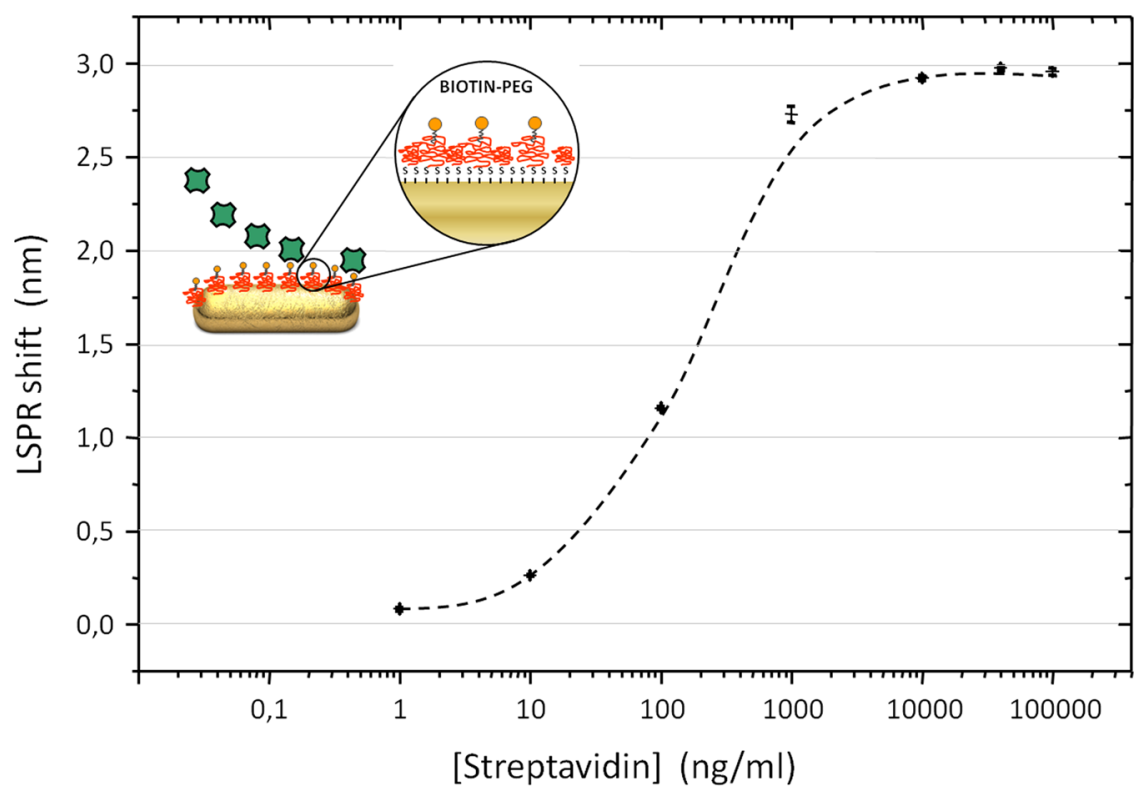

Figure 3. Parallel biosensor chip (LSPR response) for dose-dependent detection of streptavidin across all channels (zero not shown). The inset shows a schematic of the binding step in this case.

our spectrometer. The software displays separately the real-time sensograms of up to 32 individual sensors and assesses the reaction kinetics parameters. Simultaneously, the real-time response of the device is summarized in a single plot, showing the resonance shift for each of the sensing sites. This helps to control and guide the sensor preparation as well as the preview of the detection results. For more details see the video in the Supporting Information.

Preparation of the biorecognition layer is done mainly once the opto-fluidic device is assembled, with the exception of the formation of the self-assembled monolayers (SAM) which are done prior to the PDMS-substrate alignment and bonding. This step can, in principle, be also done once the chip is assembled. In Figure 2, we show two different approaches consisting of a biotin-poly(ethylene glycol) or alternatively 11mercaptoundecanoic acid monolayer. Both can be used to anchor receptors to the sensor surface in order to achieve specific detection of the corresponding target molecule. As can be seen from the schematic, both approaches allow us to detect the antigen of interest (cancer markers) either in a direct format or alternatively using a secondary polyclonal antibody in the sandwich format.

To first demonstrate the versatility of our sensing platform, we obtain a streptavidin calibration curve where we determine the sensor response as a function of streptavidin concentration in buffer by its interaction with nanoparticle arrays decorated with biotin. The streptavidin detection is a common choice for biosensing proof-of-concept tests mainly due to its extremely fast kinetics and affinity with biotin. Additionally, since one of the strategies for preparation of biorecognition layers consists of streptavidin immobilization step as a building block, from the obtained streptavidin calibration curve and reaction kinetics we can deduce the optimal parameters for the streptavidin-based antibody immobilization techniques. The opto-fluidic device is operated in two modes: buffer flows from one common inlet dividing equally into all 8 channels, or simultaneous flow from 


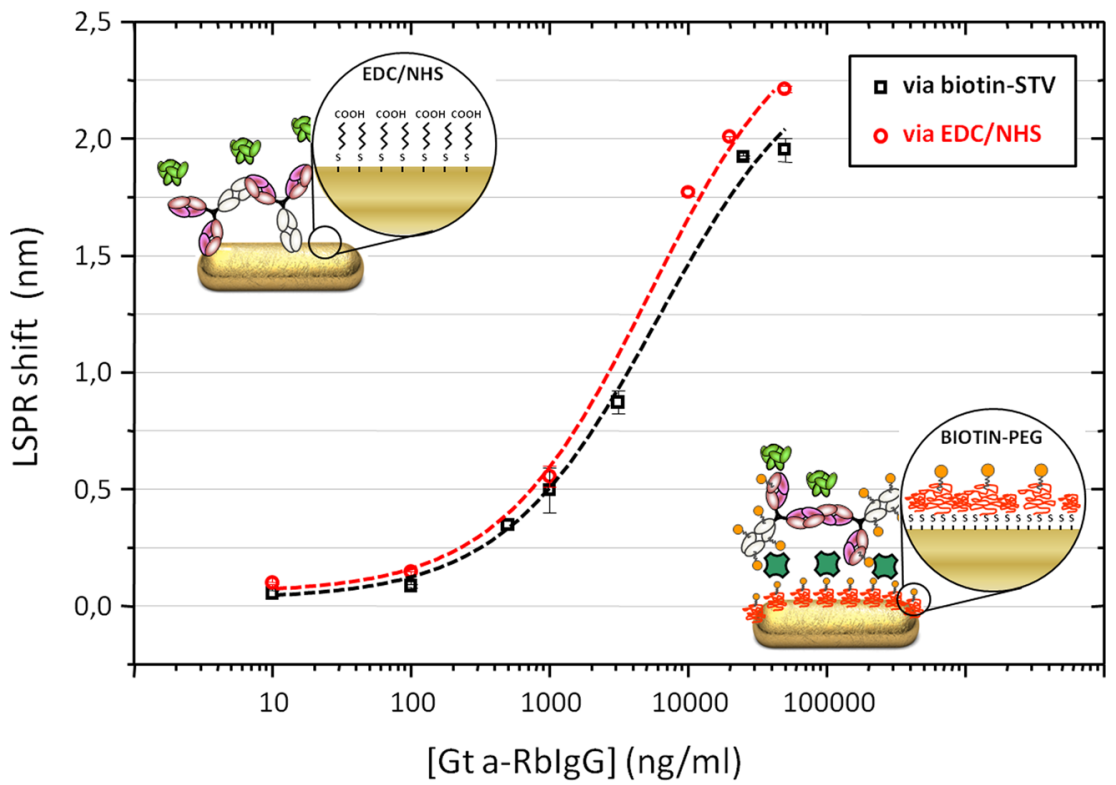

Figure 4. Comparison of two sensing approaches: Parallel biosensor chip (LSPR response) for the direct detection of model analyte in 50\% human serum by loading the sensor with an unmodified receptor using the EDC/NHS approach (left) or a biotin conjugate of the same receptor (right).

individual inlets corresponding exclusively to particular channels. These inlets are carrying various concentrations of streptavidin (from $0,1,10$ up to $100000 \mathrm{ng} / \mathrm{mL}$ ). The realtime response of 24 selected sensors is monitored, and once all displayed stable base-lines, the detection mode is engaged by opening the valves connecting individual inlets to the sensing chambers. Figure 3 displays the final complete response across all 8 channels during exposure to streptavidin solutions, established after a buffer rinse to wash off any loosely bound molecules and to establish the measurement end-line. For a demonstration of such binding, see the video in the Supporting Information.

A clear sigmoidal dose-dependence is observed with an averaged coefficient of variation $(\mathrm{CV})$ across all data points less than $1.5 \%$, displaying the highly reproducible and accurate measurement for every sensor simultaneously within the chip. The dashed line represent a sigmoidal curve fit (Hill type), which we use to conservatively define limit of detection (LOD) as the $\mathrm{EC} 10$ value. This is defined as the effective concentration to yield a $10 \%$ value of the maximum signal. Thus, the LOD as defined by this method is found to be $17 \mathrm{ng} / \mathrm{mL}$. From the realtime curves, the minimum tested concentration of $1 \mathrm{ng} / \mathrm{mL}$ is already resolved after only $900 \mathrm{~s}$, making it unnecessary to prolong the assay to the end (Figure S1 in the Supporting Information). This implies that, even lower concentrations can be detected, at the expense of the assay time. Finally, from the calibration curve, we can identify the minimum streptavidin concentration that will almost saturate the sensor surface during 900-1800 s exposure as the optimum parameters for the biorecognition receptors immobilization via avidin-biotin modification methods and in this case is $10000 \mathrm{ng} / \mathrm{mL}$.

Essential operational parameters of the sensing platform such as reproducibility and repeatability were demonstrated first with a model system using immunoglobulins (IgGs) from both rabbit and goat before moving to cancer marker detection and in particular alpha-feto-protein (AFP) and prostate specific antigen (PSA). All sensors inside the chip were saturated with the optimal concentration of streptavidin followed by a biotin conjugate of the receptor until saturation was achieved (as in the case of streptavidin). With the sensor now prepared, human serum was allowed to flow both to passivate all channel surfaces and to equilibrate the sensors for the detection of the analyte step to follow. Figure S2 in the Supporting Information summarizes the various binding events (streptavidin and biotin receptor) or passivation of the channel materials by serum and the associated LSPR reproducibility for 16 separate measurements across 8 channels. The multiplexing capability of the chip is evident, allowing for a good CV on the order of $7 \%$ or lower for the 16 measurements and also across the different stages in the sensor preparation. The averaged CV for the three steps equates to $5.4 \%$. Thus, we were confident to proceed with the analyte detection step since all sensors within the chip behaved in an analytically excellent and acceptable manner.

At this stage we investigated the response of the chip to the model analyte IgG and its ability to be detected in complex matrix, such as serum. Figure 4 shows the results of direct detection of analyte in 50\% human serum (direct detection without the sandwich step). What is initially apparent is that concentrations as low as $10-100 \mathrm{ng} / \mathrm{mL}$ of analyte could be resolved with this method. This is a level deemed clinically relevant for measuring cancer markers in serum. However, it is worth noting that, while detection of larger concentrations gives an instantaneous LSPR change, low concentrations cannot be distinguished from the "zero" control at very short analysis times as the kinetics is slowed down due to competition with the abundant serum constituents. Thus, in this case we chose $30 \mathrm{~min}$ as the appropriate time whereby we detect significant changes above the serum control for a concentration of $10 \mathrm{ng} / \mathrm{mL}$. If we again apply the Hill type sigmoidal fitting analysis, the LOD in this case (at EC10) was extracted to be $60 \mathrm{ng} / \mathrm{mL}$. We also assessed the sensor performance for the detection of IgG using an alternative strategy for immobilizing the receptor, as previously outlined in the schematic of Figure $2 \mathrm{~b}$. In this case we used the standard, well-known carbodiimide approach for linking the receptor directly to the surface without the need for prior conjugation to biotin. As a consequence, the biotin monolayer has been replaced by 11-mercaptoundecanoic acid. We used an EDC/ 


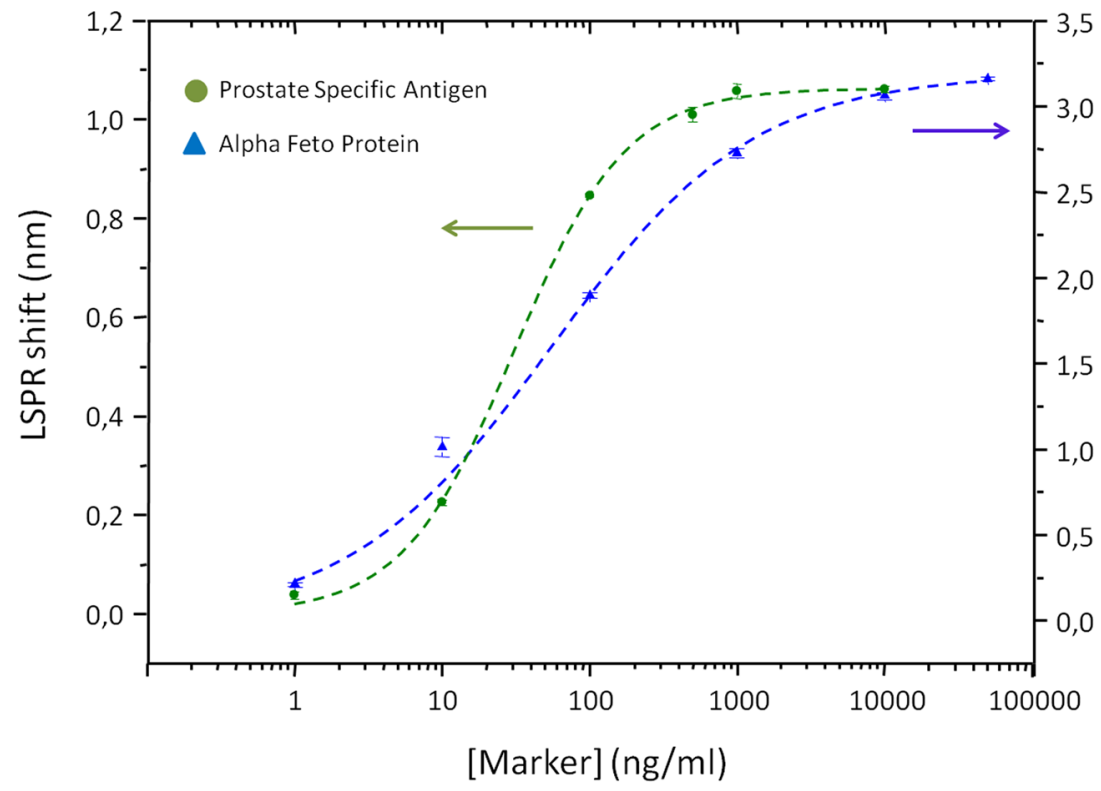

Figure 5. Parallel biosensor chip (LSPR response) for the detection of AFP and PSA cancer markers in 50\% human serum.

NHS combination for the activation of the carboxylated monolayer for the subsequent binding of the receptor (see the Supporting Information for details). By this approach we did not find any significant change in sensor performance; i.e., linear range and detection levels were on the same order with an excellent coefficient of variation noted. Fitting of both these curves with the usual Hill-type sigmoid shows excellent fitting to the data with both coefficient of determination ( $R$ squared) values greater than 0.998 (0.9989 vs 0.9993$)$. This high goodness of fitting further highlights the compatibility of our microfluidic chip to various sensing strategies and chemical approaches. The real-time curves for the individual concentrations can be seen in Figure S3 of the Supporting Information.

To test our LSPR sensing platform in the frame of cancer diagnostic and treatment follow-up, we investigate two different clinically relevant molecules, such as AFP and PSA. In men, nonpregnant women, and children, AFP $(70 \mathrm{kDa})$ in the blood can mean certain types of cancer, especially that cancer of the testicles, ovaries, stomach, pancreas, or liver is present. High levels of AFP may also be found in Hodgkin's disease, lymphoma, brain tumors, and renal cell cancer. In most cases the levels in affected patients are in the $500-1000 \mathrm{ng} / \mathrm{mL}$ range. ${ }^{33,34}$ The prostate-specific antigen is a $34 \mathrm{kDa}$ glycoprotein produced almost exclusively by the prostate gland and is a member of the kallikrein-related peptidase family. It is present in small quantities in the serum of men with healthy prostates but is often elevated in the presence of prostate cancer or other prostate disorders. ${ }^{35}$ PSA is not a unique indicator of prostate cancer but may also detect prostatitis or benign prostatic hyperplasia. ${ }^{36}$ In the United States, the U.S. Food and Drug Administration has approved the PSA test for annual screening of prostate cancer in men of age 50 and older. PSA levels between 4 and $10 \mathrm{ng} / \mathrm{mL}$ are considered to be suspicious and consideration should be given to confirming the abnormal PSA with a repeat test or alternative confirmatory analysis.

In Figure 5, we used the EDC/NHS approach to simplify the methodology and avoid prior conjugation of antibody receptors with biotin linkers, in order not to jeopardize affinity. Thus, the antibody receptor is directly linked to an 11-mercaptoundecanoic acid prepared monolayer on the gold arrays; cancer markers at various concentrations are injected and monitored in $50 \%$ serum, and detection is completed by using secondary polyclonal antibodies. First, considering AFP (blue curve), we can clearly measure small variations in concentration of AFP in $50 \%$ human serum well below the clinically significant level $(500 \mathrm{ng} / \mathrm{mL})$. The smallest discernible concentration we could measure above the background was deemed to be $500 \mathrm{pg} / \mathrm{mL}$, which represents picomolar levels $\left(10^{-12} \mathrm{M}\right)$ of AFP (Figure S4 in the Supporting Information). The linear range (EC20-EC80) of this sensor is wide $(5-1000 \mathrm{ng} / \mathrm{mL})$, and as can be seen from the data for each concentration the averaged coefficient of variation across all concentrations is very low (2.4\%) showing a very reproducible measurement for up to three independent sensors per channel. Fitting of the data also returns a very acceptable $R$ squared value of 0.998 , further highlighting the repeatability and reproducibility of this parralel approach for measuring a complete working range of any specific analyte of interest simultaneously.

To further illustrate the universal nature of our biosensing platform for detection of alternative markers, we also demonstrate (green curve) the complete detection of prostate specific antigen and the ability to quickly detect analyte in the $\mathrm{ng} / \mathrm{mL}$ range in a matter of minutes with excellent reproducibility (Figure S5 in the Supporting Information). The linear range for PSA can be determined to be lying between 10 and $100 \mathrm{ng} / \mathrm{mL}$, again based on the EC20-EC80 parameter. A Hill type fitting of the data reveals an excellent $R$ squared value of 0.9994 , and this is coupled to an excellent coefficient of variation for each point with the averaged $\mathrm{CV}$ of $1.3 \%(n=18)$. The lowest concentration attainable was found to be $1 \mathrm{ng} / \mathrm{mL}$, which gives a sufficient signal above the background level. To shift the LOD to lower concentrations, it is possible to load the surface of the sensor with a lower quantity of receptor. However, we observed a decrease in absolute signal and a slight modification of the linear range. Therefore, a proper optimization of this parameter is necessary for each particular case according to the level of concentrations needed to be detected. Nevertheless, this current level of 
sensitivity shown by the PSA sensor is deemed suitable for detecting serum PSA since affected patients have levels greater than $4 \mathrm{ng} / \mathrm{mL}(4-20 \mathrm{ng} / \mathrm{mL})$ range and we clearly can measure sufficient LSPR changes for such concentrations. The combined detection of both AFP and PSA can be seen in Figure $\mathrm{S} 6$ in the Supporting Information.

This work highlights the synergy of many scientific disciplines such as plasmonics, micro- and nanofabrication, microfluidics, optics, surface chemistry, and immunology. It is through the amalgamation of all of these fields that we have presented for the first time a functional LSPR-based device that can answer many of the contemporary market demands. The generic nature of this biosensing platform allows for parallel analyses of cancer markers in a rapid, automated, and accurate manner and all under simple 50\% dilution of the serum sample. The microfluidic degree of integration could also be up-scaled to achieve higher throughput and make the device suitable for multiple marker, simultaneous calibration, and detection within a single chip, thus fulfilling a true lab-on-a-chip or total analysis system ( $\mu$ TAS) criteria.

\section{ASSOCIATED CONTENT}

\section{S Supporting Information}

Supporting video files and additional discussions including the details of the fabrication, characterization, microfluidics, and sensing methods. This material is available free of charge via the Internet at http://pubs.acs.org.

\section{AUTHOR INFORMATION}

\section{Corresponding Authors}

*E-mail: (M.P.K.) mark.kreuzer@icfo.es.

*E-mail: (R.Q.) romain.quidant@icfo.es.

\section{Author Contributions}

S.S.A. and M.A.O. contributed equally to this work. S.S.A., M.P.K., M.A.O., and V.S. devised and performed the experiments. J.B., J.R., and S.S.A. built the optical setup. J.B. and S.S.A. wrote and tested the labview software. J.-L.G.C. and S.J.M. designed the microfluidic chip. S.S.A. and J.-L.G.C. manufactured the microfluidic chips. M.P.K., S.S.A., and R.Q. wrote the manuscript. All authors read and commented on the final manuscript.

\section{Notes}

The authors declare no competing financial interest.

\section{ACKNOWLEDGMENTS}

The authors thank Dr. Mathieu L. Juan for help on the initial versions of the Labview software control. This work was supported by the European Commission's Seventh Framework Programme under Grants SPEDOC (248835), ERC-Plasmolight (259196), and Fundació Privada CELLEX.

\section{REFERENCES}

(1) Anker, J. N.; Hall, W. P.; Lyandres, O.; Shah, N. C.; Zhao, J.; Van Duyne, R. P. Nat. Mater. 2008, 7, 442-453.

(2) Yonzon, C. R.; Jeoung, E.; Zou, S.; Schatz, G. C.; Mrksich, M.; Van Duyne, R. P. J. Am. Chem. Soc. 2004, 126, 12669-12676.

(3) Svedendahl, M.; Chen, S.; Dmitriev, A.; Kall, M. Nano Lett. 2009, 9, 4428-4433.

(4) Raether, H. Surface plasmons on smooth surfaces; Springer: Berlin, 1988; Chapter 2, pp 4-39.

(5) Homola, J. Surface plasmon resonance based sensors; SpringerVerlag: Berlin, 2006; Vol. 4.
(6) Sebba, D. S.; Watson, D. A.; Nolan, J. P. ACS Nano 2009, 3, $1477-1484$

(7) Ament, I.; Prasad, J.; Henkel, A.; Schmachtel, S.; Sönnichsen, C. Nano Lett. 2012, 12, 1092-1095.

(8) Zijlstra, P.; Paulo, P. M.; Orrit, M. Nat. Nanotechnol. 2012, 7, 379-382.

(9) Al Balushi, A. A.; Zehtabi-Oskuie, A.; Gordon, R. Biomed. Opt. Exp. 2013, 4, 1504-1511.

(10) Ruemmele, J. A.; Hall, W. P.; Ruvuna, L. K.; Van Duyne, R. P. Anal. Chem. 2013, 85, 4560-4566.

(11) Dahlin, A. B. Sensors 2012, 12, 3018-3036.

(12) Gerion, D.; Day, G. J. Bio. Pharm. Int. 2010, 23, 38-45.

(13) Chen, S.; Svedendahl, M.; Van Duyne, R. P.; Käll, M. Nano Lett. 2011, 11, 1826-1830.

(14) Rodríguez-Lorenzo, L.; de La Rica, R.; Álvarez-Puebla, R. A.; Liz-Marzán, L. M.; Stevens, M. M. Nat. Mater. 2012, 11, 604-607.

(15) de La Rica, R.; Stevens, M. M. Nat. Nanotechnol. 2012, 7, 821824.

(16) Haynes, C. L.; Van Duyne, R. P. J. Phys. Chem. B 2001, 105, 5599-5611.

(17) Fredriksson, H.; Alaverdyan, Y.; Dmitriev, A.; Langhammer, C.; Sutherland, D. S.; Zäch, M.; Kasemo, B. Adv. Mater. 2007, 19, 42974302.

(18) Lee, S. W.; Lee, K. S.; Ahn, J.; Lee, J. J.; Kim, M. G.; Shin, Y. B. ACS Nano 2011, No. 5, 897-904.

(19) Jain, P. K.; El-Sayed, M. A. Nano Lett. 2008, 8, 4347-4352.

(20) Aćimović, S. S.; Kreuzer, M. P.; González, M. U.; Quidant, R. ACS Nano 2009, 3, 1231-1237.

(21) Lassiter, J. B.; Sobhani, H.; Fan, J. A.; Kundu, J.; Capasso, F.; Nordlander, P.; Halas, N. J. Nano Lett. 2010, 10, 3184-3189.

(22) Cetin, A. E.; Altug, H. ACS Nano 2012, 6, 9989-9995.

(23) Raschke, G.; Brogl, S.; Susha, A. S.; Rogach, A. L.; Klar, T. A.; Feldmann, J.; Fieres, B.; Petkov, N.; Bein, T.; Nichtl, A.; Kürzinger, A. Nano Lett. 2004, 4, 1853-1857.

(24) Tam, F.; Moran, C.; Halas, N. J. Phys. Chem. B 2004, 108, 17290-17294.

(25) Eftekhari, F.; Escobedo, C.; Ferreira, J.; Duan, X.; Girotto, E. M.; Brolo, A. G.; Gordon, R.; Sinton, D. Anal. Chem. 2009, 81, 43084311.

(26) Jonsson, M. P.; Dahlin, A. B.; Feuz, L.; Petronis, S.; Höök, F. Anal. Chem. 2010, 82, 2087-2094.

(27) Yanik, A. A.; Huang, M.; Artar, A.; Chang, T. Y.; Altug, H. Appl. Phys. Lett. 2010, 96, 021101-021101.

(28) Chen, S.; Svedendahl, M.; Käll, M.; Gunnarsson, L.; Dmitriev, A. Nanotechnology 2009, 20, 434015.

(29) Unger, M. A.; Chou, H. P.; Thorsen, T.; Scherer, A.; Quake, S. R. Science 2000, 288, 113-116.

(30) Thorsen, T.; Maerkl, S. J.; Quake, S. R. Science 2002, 298, 580584.

(31) Dahlin, A. B.; Tegenfeldt, J. O.; Höök, F. Anal. Chem. 2006, 78, $4416-4423$

(32) Dahlin, A. B.; Chen, S.; Jonsson, M. P.; Gunnarsson, L.; Käll, M.; Höök, F. Anal. Chem. 2009, 81, 6572-6580.

(33) http://www.medicinenet.com/alpha-fetoprotein_blood_test/ page2.htm.

(34) http://en.wikipedia.org/wiki/Alpha-fetoprotein.

(35) Catalona, W. J.; Richie, J. P.; Ahmann, F. R.; Hudson, M. A.; Scardino, P. T.; Flanigan, R. C.; Dekernion, J. B.; Ratliff, T. L.; Kavoussi, L. R.; Dalkin, B. L. J. Urol. 1994, 151, 1283-1290.

(36) Velonas, V. M.; Woo, H. H.; Remedios, C. G.; Assinder, S. J. Int. J. Mol. Sci. 2013, 14, 11034-60.

(37) Garcia-Cordero, J. L.; Maerkl, S. J. Lab Chip 2014, DOI: $10.1039 /$ C3LC51153G. 